\title{
MÉTODOS DE PREPARO DO SOLO: ALTERAÇÕES NA RUGOSIDADE DO SOLO ${ }^{1}$
}

\section{ALBERTO CARVALHO FILHO ${ }^{2}$, JOSÉ F. CENTURION ${ }^{3}$, ROUVERSON P. DA SILVA $^{4}$, CARLOS E. A. FURLANI ${ }^{5}$, LUÍS C. C. CARVALHO ${ }^{6}$}

RESUMO: Procurando relacionar os métodos de preparo de solo com alguns dos efeitos em um Latossolo Vermelho distrófico, foi conduzido, no município de Uberaba - MG, um trabalho utilizando-se do delineamento experimental em blocos casualizados, com cinco tratamentos e quatro repetições, tendo como tratamentos os preparos de solo: escarificador, enxada rotativa, arado de aivecas, grade aradora e arado de discos. Foram avaliadas a área mobilizada do solo e a área de elevação, tendo-se como referência a superfície natural do solo antes da mobilização, o empolamento do solo, a espessura média da camada mobilizada, o índice de rugosidade e a modificação da rugosidade do solo para a comparação da rugosidade antes e após sua mobilização. O escarificador proporcionou menor empolamento e maior índice de rugosidade do solo, enquadrando-se como preparo conservacionista por desestruturar menos o solo, podendo colaborar com as menores perdas de solo por erosão.

PALAVRAS-CHAVE: área de elevação, área mobilizada, índice de rugosidade.

\section{SOIL TILLAGE METHODS: ALTERATIONS IN THE ROUGHNESS OF THE SOIL}

ABSTRACT: Trying to relate the soil tillage methods with some of their effects in Haplustox, it was installed in the municipal district of Uberaba/MG, a research using a completely randomized experimental design in factorial array with five treatments and four replications. The treatments were composed by the soil tillage: chisel plow, rotary tiller, moldboard plow, disk harrow and disk plow. They were appraised: soil mobilized area, elevation area, medium thickness of the mobilized layer, roughness index and the alteration of the roughness of the soil. It was verified that the chisel plow provided larger roughness index of the soil being framed in the conservationist method disaggregating less the soil, collaborating with the smallest soil losses for erosion.

KEYWORDS: area of soil elevation, mobilized area, roughness index.

\section{INTRODUÇÃO}

Muitos sistemas de exploração agrícola têm proporcionado ao solo acelerado processo de degradação, com desequilíbrio de suas características físicas, químicas e biológicas, afetando o seu potencial produtivo.

Para cada condição de solo e operação agrícola, existe um equipamento adequado. O solo deve ser preparado com o mínimo de mobilização, não implicando, com isso, diminuição da profundidade de operação, mas sim redução do número de operações, deixando rugosa a superfície do solo e mantendo os resíduos culturais, total ou parcialmente, sobre a superfície, trazendo benefícios para a sustentabilidade ambiental e também, muitas vezes, maior economia. Observa-se, entretanto, que a maior parte dos equipamentos utilizados na mobilização do solo não atende a algumas dessas condições tidas como ideais.

\footnotetext{
${ }^{1}$ Extraído da Dissertação de Mestrado do primeiro autor junto à UNESP, Jaboticabal - SP.

${ }^{2}$ Prof. Dr., FAZU, Uberaba - MG e FAFRAM, Ituverava - SP, alberto@ fazu.br, alberto@ feituverava.com.br

${ }^{3}$ Prof. Adjunto, Departamento de Solos e Adubos, UNESP - Jaboticabal - SP.

${ }^{4}$ Prof. Dr., Departamento de Engenharia Rural, UNESP - Jaboticabal - SP. Bolsista de Produtividade CNPq.

${ }^{5}$ Prof. Adjunto, Departamento de Engenharia Rural, UNESP - Jaboticabal - SP. Bolsista de Produtividade CNPq.

${ }^{6}$ Graduando em Agronomia, UFLA, Lavras - MG.

Recebido pelo Conselho Editorial em: 23-11-2005

Aprovado pelo Conselho Editorial em: 19-3-2007
} 
O sistema convencional de preparo do solo consiste na realização de uma aração, caracterizado pelo preparo primário do solo, seguida de duas gradagens para destorroamento e nivelamento (DERPSCH et al., 1991), também denominada de operações de preparo periódico secundário. O emprego desse mesmo manejo, ao longo de vários anos, poderá proporcionar a desestruturação da superfície dos solos, deixando-os mais suscetíveis ao processo de erosão e à formação de impedimentos mecânicos logo abaixo das camadas de solo movimentadas pelos equipamentos, os quais podem interferir no desenvolvimento radicular das culturas, acarretando redução na produtividade (BAUDER et al., 1981).

De acordo com ASAE (1982) e DALLMEYER (1994), o preparo mínimo ou reduzido do solo pode ser definido como aquele que proporciona menor número de operações que o preparo convencional, resultando em menor incorporação de resíduos vegetais, menor inversão do solo, menor custo de preparo e redução das perdas de solo e água por erosão.

Considera-se como preparo conservacionista aquele que proporciona a menor mobilização possível do solo, visando a preservar sua estruturação, mantendo no mínimo $30 \%$ da superfície do solo coberta com resíduos culturais, entre o período compreendido da colheita da cultura anterior e a implantação da cultura seguinte (GROHMANN \& ARRUDA, 1961 e BUHLER, 1995). Assim, pode-se considerar que tanto o preparo reduzido quanto o sistema de semeadura direta, que é um tipo de preparo na linha com mínima mobilização do solo, podem ser enquadrados como preparo conservacionista.

A avaliação dos perfis mobilizados pelos preparos do solo pode oferecer informações importantes. Segundo DANIEL \& MARETTI (1990), dois são os fenômenos decorrentes da operação de preparo periódico: o deslocamento vertical do perfil do solo e a sua área mobilizada, devendo ser realizados levantamentos de três perfis: o perfil da superfície natural, da superfície de elevação e o perfil interno do solo mobilizado. A avaliação das áreas entre os perfis pode ser determinada por meio de gráficos, utilizando-se de técnicas de planimetria ou programas computacionais. FURLANI (2000), avaliando três sistemas de preparo do solo (convencional, escarificação e plantio direto), observou que a superfície do solo, após as operações de preparo, semeadura, cultivos e chuvas, voltou a sua condição original, destacando que, durante a safra da cultura, tem-se aos poucos o retorno à condição original.

Segundo KAMPHORST et al. (2000), pode-se expressar a rugosidade superficial do solo por diversos índices. GAMERO \& BENEZ (1990), SANTOS (1993), COAN (1995) e OLIVEIRA (1997) sugerem que a avaliação da rugosidade superficial de um solo pode ser feita por meio da utilização do índice de rugosidade proposto por ALLMARAS et al. (1966). A rugosidade do solo deve ser medida, também, após o preparo. Dessa maneira, pode-se determinar o índice de rugosidade da superfície mobilizada.

O índice de modificação da rugosidade expressa, em termos percentuais, a mudança entre a rugosidade inicial e a rugosidade final, após o preparo do solo, ou seja, é a rugosidade resultante da ação dos equipamentos em relação ao estado natural em que o solo se encontrava. Dessa forma, solos mais rugosos podem tornar-se mais uniformes após a ação de certos equipamentos, bem como solos com superfície mais plana podem tornar-se mais rugosos.

O preparo do solo é o método mais usual para modificar a rugosidade do solo. Quando o solo é submetido a um sistema conservacionista, onde o preparo é realizado com escarificador, seguido ou não de gradagem, a superfície do solo apresenta-se mais rugosa do que quando submetida ao sistema convencional com arados e grades (BERTOL et al., 2006). No entando, o sistema convencional pode apresentar uma superfície mais rugosa comparado ao solo mantido sem preparo por algum tempo, como é o caso do plantio direto (SCHICK et al., 2000).

O aumento da rugosidade superficial, em função do preparo do solo, foi explicado por BERTOL et al. (2006) devido à elevação da superfície do solo acima do nível original, criando espaços porosos internos no solo e na superfície (microdepressões), além de microelevações superficiais. 
A rugosidade superficial do solo induzida por métodos de preparo é requerimento importante nos sistemas de manejo de caráter conservacionista. Isso se deve ao fato de que aumenta a retenção e a infiltração superficial da água no solo, reduz a velocidade e o volume do escoamento superficial, aprisionando os sedimentos da erosão e diminuindo danos causados pela erosão hídrica (CASTRO et al., 2006).

Na agricultura moderna, o preparo periódico do solo é considerado uma operação básica e, nas últimas décadas, vem passando por considerável evolução. Nos últimos anos, a atenção está voltada para sistemas de semeadura direta e de preparo reduzido ou de cultivo mínimo do solo, ou seja, para aqueles cujas operações resultem em menor consumo de energia, que visem à conservação do solo e da água e não causem prejuízos no desenvolvimento e na produtividade das plantas (COAN, 1995).

Este trabalho teve como objetivo avaliar a área mobilizada, a área de elevação da superfície do solo, o empolamento do solo, a espessura média da camada mobilizada, o índice de rugosidade e a modificação da rugosidade do solo ocasionados pela mobilização promovida pelo escarificador, enxada-rotativa, arado de aivecas, grade aradora e arado de discos em um Latossolo Vermelho distrófico, textura média, em relevo plano.

\section{MATERIAL E MÉTODOS}

O trabalho foi desenvolvido na Área Experimental da FAZU - Faculdades Associadas de Uberaba - MG, com as coordenadas geográficas, longitude: $47^{\circ} 57^{\prime} \mathrm{W}$, latitude: $19^{\circ} 44^{\prime} \mathrm{S}$ e altitude de $780 \mathrm{~m}$. O clima de Uberaba, segundo a classificação de Köeppen, é do tipo Aw, tropical quente úmido, com inverno frio e seco. As médias anuais de precipitação e temperatura são de $1.474 \mathrm{~mm}$ e $22,6^{\circ} \mathrm{C}$, respectivamente.

O experimento foi instalado em 1999, em um Latossolo Vermelho distrófico, textura média, relevo plano (EMBRAPA, 1999) e densidade média na camada de 0 a $20 \mathrm{~cm}$ igual a $1,73 \mathrm{~g} \mathrm{~cm}^{-3}$. O delineamento utilizado foi em blocos casualizados, com cinco tratamentos e quatro repetições, resultando num total de 20 parcelas, tendo cada parcela $100 \mathrm{~m}^{2}(10 \times 10 \mathrm{~m})$. Os tratamentos foram constituídos pelo preparo periódico do solo efetuados com: a) escarificador, modelo AST 5/5, montado, marca Tatu-Marchesan, desprovido de rolo destorroador, equipado com cinco hastes espaçadas de $39 \mathrm{~cm}$, largura de corte de $156 \mathrm{~cm}$ e massa de $390 \mathrm{~kg}$; b) enxada rotativa montada, equipada com seis flanges, apresentando quatro lâminas tipo veloz por flange, operando a $172 \mathrm{rpm}$, com a placa de impacto abaixada, largura de corte de $90 \mathrm{~cm}$ e massa total de $220 \mathrm{~kg}$; c) arado de aivecas fixas, montado, modelo AAP, marca Tatu-Marchesan, equipado com duas aivecas recortadas, largura de corte de $90 \mathrm{~cm}$ e massa de $415 \mathrm{~kg}$; d) grade aradora de arrasto, excêntrica, modelo GAM 14, marca Tatu-Marchesan, equipada com 14 discos recortados de $61 \mathrm{~cm}$ (24"), espaçados de $23 \mathrm{~cm}$, largura de corte de $150 \mathrm{~cm}$ e massa de $1.210 \mathrm{~kg}$, e e) arado de discos fixos, montado, modelo AF 4, marca Tatu-Marchesan, equipado com quatro discos de $66 \mathrm{~cm}$ (26") de diâmetro, espaçados de $57 \mathrm{~cm}$, largura de corte de $123 \mathrm{~cm}$ e massa de $492 \mathrm{~kg}$.

Utilizou-se do trator Ford 6610, 4X2, com potência de $63 \mathrm{~kW}(86 \mathrm{cv})$, operando com velocidade média de $5 \mathrm{~km} \mathrm{~h}^{-1}$, na tração dos arados, do escarificador e da grade, e um microtrator Agrale 4100, 4X2, com potência de 10,8 kW (15 cv), e velocidade média de operação de $1,5 \mathrm{~km} \mathrm{~h}^{-}$ 1 , para tracionar a enxada rotativa.

Os arados de discos, de aivecas e o escarificador foram regulados para cortarem o solo à profundidade média de $20 \mathrm{~cm}$, a grade aradora a $13 \mathrm{~cm}$ e a enxada rotativa a $8 \mathrm{~cm}$.

A grade aradora e a enxada rotativa estavam reguladas para a obtenção de máxima profundidade de corte, o mesmo não ocorrendo para os demais equipamentos avaliados.

O preparo convencional foi efetuado com os arados e com a grade aradora, sendo realizada a primeira gradagem de destorroamento e nivelamento logo após o preparo primário, e a segunda, antecedendo a semeadura com grade niveladora marca Piccin, de 32 discos de 18 polegadas, com 
massa aproximada de $1.200 \mathrm{~kg}$. Com o escarificador, efetuou-se o preparo reduzido do solo, sendo realizada apenas uma gradagem de destorroamento-nivelamento, antecedendo a semeadura, e com a enxada rotativa, o solo não sofreu nova mobilização.

A umidade gravimétrica do solo no momento do preparo foi de $16 \mathrm{~g} \mathrm{~kg}^{-1}$. As amostras de solo foram retiradas por meio de trado tipo holandês, em três pontos por parcela, na profundidade a ser mobilizada por cada equipamento.

Na obtenção do índice de rugosidade e do perfil mobilizado do solo, que permite calcular a área mobilizada, a área de elevação e o empolamento do solo, utilizou-se de um perfilômetro com largura útil de $250 \mathrm{~cm}$, com pontos tomados de $5 \mathrm{em} 5 \mathrm{~cm}$, perfazendo um total de 50 leituras, obtidas por meio de uma régua de $1 \mathrm{~m}$ de comprimento, graduada de $1 \mathrm{em} 1 \mathrm{~cm}$, conforme adaptação realizada do trabalho descrito por GAMERO \& BENEZ (1990). O perfilômetro foi instalado sobre uma base previamente nivelada, montada na direção transversal ao deslocamento do trator. Após a obtenção das 50 leituras, o perfilômetro foi deslocado na direção longitudinal, fazendo-se coincidir o ponto da última leitura da posição anterior com a primeira leitura no novo posicionamento. Esse procedimento foi repetido até a obtenção de 200 leituras, conforme indicação feita por ALLMARAS et al. (1966). Assim, foram realizadas as leituras do perfil do solo antes dos preparos, obtendo-se o perfil natural, e após o preparo do solo, para obtenção do perfil da superfície do solo após mobilizado e o perfil interno do solo mobilizado, denominados, respectivamente, de perfil de elevação e perfil de fundo. Esse último foi obtido após a retirada do solo mobilizado pelo equipamento.

Os cálculos da área de elevação e da área mobilizada foram obtidos por meio da Regra de Simpson [eq. (1)]:

$$
\int_{x_{0}}^{x_{n}} d x=\frac{h}{3}\left(f_{0}+4 f_{1}+2 f_{2}+4 f_{3}+2 f_{4}+\ldots+2 f_{n-2}+4 f_{n-1}+f n\right)
$$

em que,

$$
\mathrm{h}=\frac{\mathrm{X}_{\mathrm{n}}-\mathrm{X}_{\mathrm{o}}}{\mathrm{n}}, \quad \mathrm{X}_{\mathrm{n}}>\mathrm{X}_{\mathrm{o}}
$$

sendo,

n - número de intervalos;

$\mathrm{f}$ - altura das cotas, $\mathrm{mm}$;

h - distância entre cotas, $\mathrm{cm}$, e

$\mathrm{X}$ - número de cotas.

O índice de rugosidade superficial [eq.(2)] representa o produto do desvio-padrão entre os logaritmos naturais das leituras das elevações, pela altura média das elevações (ALLMARAS et al., 1966). em que,

$$
\sigma y=\sigma x h m
$$

$\sigma y$ - estimativa do índice de rugosidade representada pelo desvio-padrão entre as alturas, mm;

$\sigma x$ - desvio-padrão entre os logaritmos naturais das alturas, e

$\mathrm{hm}$ - média das alturas, $\mathrm{mm}$.

A área de solo mobilizada consiste na área situada entre o perfil original e o perfil de fundo de sulco, enquanto a área de elevação é aquela situada entre o perfil original e o perfil da superfície do solo após a mobilização, conforme descrito por GAMERO \& BENEZ (1990).

Obtidos os dados do perfil do solo mobilizado, a espessura média foi calculada por meio da eq.(3):

$$
\mathrm{Ec}=\frac{\mathrm{Am}}{\mathrm{Cp}}
$$


em que,

Ec - espessura média da camada mobilizada, m;

Am - área mobilizada do solo, $\mathrm{m}^{2}$, e

$\mathrm{Cp}$ - comprimento do perfilômetro $\mathrm{m}$.

O empolamento do solo [eq.(4)] foi determinado pela razão entre a área de elevação e a área mobilizada pelos órgãos ativos do equipamento, segundo GAMERO \& BENEZ (1990).

$$
\mathrm{Em}=\frac{\mathrm{Ae}}{\mathrm{Am}} 100
$$

em que,

Em - empolamento, \%;

Ae - área de elevação, $\mathrm{m}^{2}$, e

Am - área mobilizada, $\mathrm{m}^{2}$.

A modificação da rugosidade do solo [eq.(5)] foi obtida considerando-se a diferença entre os índices de rugosidade medidos após e antes do preparo, em relação ao índice de rugosidade antes do preparo do solo, expressa em percentagem (SANTOS, 1993).

$$
\mathrm{MR}=\frac{\mathrm{IRf}-\mathrm{IRi}}{\mathrm{IRi}} 100
$$

em que,

MR - modificação da rugosidade, \%;

IRf - índice de rugosidade após o preparo do solo, e

IRi - índice de rugosidade antes do preparo do solo.

Após a análise de variância, quando significativa, foi aplicado o teste de Tukey para comparação de médias dos tratamentos.

\section{RESULTADOS E DISCUSSÃO}

Os valores médios referentes à área mobilizada para cada equipamento encontram-se na Tabela 1 e mostram-se diferentes estatisticamente.

TABELA 1. Área mobilizada (Am), área de elevação (Ae), espessura média da camada mobilizada (Emc), empolamento (E), índice de rugosidade e modificação da rugosidade do solo (MR) em função do método de preparo periódico.

\begin{tabular}{lccrcccr}
\hline \multicolumn{1}{c}{$\begin{array}{c}\text { Método de } \\
\text { Preparo }\end{array}$} & $\begin{array}{c}\mathrm{Am} \\
\left(\mathrm{m}^{2}\right)\end{array}$ & $\begin{array}{c}\mathrm{Ae} \\
\left(\mathrm{m}^{2}\right)\end{array}$ & $\begin{array}{c}\text { Emc } \\
(\mathrm{cm})\end{array}$ & $\begin{array}{c}\mathrm{E} \\
(\%)\end{array}$ & \multicolumn{2}{c}{ Índice de Rugosidade do Solo $(\mathrm{cm})$} & $\begin{array}{c}\text { MR } \\
(\%)\end{array}$ \\
\hline Escarificador & $0,49 \mathrm{~b}^{1}$ & $0,10 \mathrm{a}$ & $19,8 \mathrm{~b}$ & $19,6 \mathrm{~b}$ & $2,01 \mathrm{a}$ & $3,87 \mathrm{ab}$ & $116,9 \mathrm{a}$ \\
Enxada rotativa & $0,20 \mathrm{c}$ & $0,08 \mathrm{ab}$ & $8,0 \mathrm{c}$ & $40,9 \mathrm{a}$ & $2,20 \mathrm{a}$ & $2,56 \mathrm{c}$ & $28,9 \mathrm{a}$ \\
Arado de aivecas & $0,60 \mathrm{a}$ & $0,07 \mathrm{~b}$ & $23,8 \mathrm{a}$ & $10,9 \mathrm{c}$ & $2,25 \mathrm{a}$ & $4,29 \mathrm{a}$ & $126,5 \mathrm{a}$ \\
Grade aradora & $0,27 \mathrm{c}$ & $0,06 \mathrm{~b}$ & $10,5 \mathrm{c}$ & $24,3 \mathrm{~b}$ & $2,41 \mathrm{a}$ & $3,11 \mathrm{bc}$ & $41,0 \mathrm{a}$ \\
Arado de discos & $0,48 \mathrm{~b}$ & $0,09 \mathrm{a}$ & $19,4 \mathrm{~b}$ & $18,0 \mathrm{bc}$ & $2,01 \mathrm{a}$ & $4,35 \mathrm{a}$ & $141,4 \mathrm{a}$ \\
C.V.(\%) & 9,5 & 13,6 & 9,6 & 14,8 & 24,9 & 9,8 & 59,1 \\
\hline
\end{tabular}

${ }^{1}$ Médias seguidas pela mesma letra, na coluna, são iguais estatisticamente, a 5\% de probabilidade, pelo teste de Tukey.

Observa-se que os arados e o escarificador apresentaram maior capacidade de penetração e mobilização do solo. Destaca-se o arado de aivecas com $0,60 \mathrm{~m}^{2}$, seguido pelo escarificador com $0,49 \mathrm{~m}^{2}$ e pelo arado de discos com $0,48 \mathrm{~m}^{2}$. Conforme também observado por COAN (1995), as áreas mobilizadas pela grade aradora e a enxada rotativa foram aproximadamente a metade daquelas obtidas com os demais equipamentos $\left(0,27 \mathrm{~m}^{2}\right.$ para a grade e $0,20 \mathrm{~m}^{2}$ para a enxada rotativa). Isso é justificado por apresentarem seus órgãos ativos fixados em um mesmo eixo, característica que dificulta a penetração no solo. Entretanto, SALVADOR et al. (1993), 
trabalhando com modelos distintos e provavelmente em velocidades diferentes, mostraram que os preparos envolvendo arado de discos proporcionaram menor área mobilizada do que os preparos com grade aradora e escarificador, justificado pelo arado possuir menor largura efetiva de corte que os demais equipamentos, embora todos estivessem regulados para atuarem à mesma profundidade.

Apesar de não existir diferença significativa entre os valores obtidos para o arado de discos e o escarificador, deve-se considerar a maior dificuldade de penetração dos discos, comparados às hastes parabólicas dos escarificadores.

A avaliação desse parâmetro deve ser bastante criteriosa, considerando-se que a ação de cada equipamento no solo é diferente para áreas mobilizadas semelhantes. Assim, para o enterrio de plantas invasoras, os arados são preferidos, destacando-se o arado de aivecas; para a incorporação mais homogênea de corretivos e fertilizantes, na profundidade mobilizada, são preferidas a grade aradora e a enxada rotativa.

$\mathrm{Na}$ Tabela 1, indica-se também haver efeitos de diferentes equipamentos sobre a área de elevação do solo, devido aos órgãos ativos possuírem características diferentes quanto à capacidade de penetração, corte, elevação e inversão da leiva.

A grade aradora apresentou menor área de elevação do solo $\left(0,06 \mathrm{~m}^{2}\right)$, justificada pelos seus discos estarem dispostos em forma de secções, o que limita a obtenção de maiores profundidades de operação, resultando em menor volume de solo elevado, ao contrário da enxada rotativa, com $0,08 \mathrm{~m}^{2}$ de área de elevação e que também possui seus órgãos ativos em forma de secção, limitando atingir maiores profundidades, mas equipada com lâminas do tipo veloz e com o anteparo traseiro levantado. SILVA \& GAMERO (1993) e COAN (1995) observaram, também, desempenho semelhante para esses equipamentos durante seus estudos, ao contrário de SALVADOR et al. (1993), que não encontraram diferenças significativas entre os valores de área de elevação para os arados, grades e escarificadores.

Observa-se que houve efeito dos tratamentos na espessura média da camada mobilizada (Tabela 1), em que o arado de aivecas com 23,8 cm, o escarificador com 19,8 cm e o arado de discos com 19,4 cm, possuem maior capacidade de penetração no solo, comparados à grade aradora e à enxada rotativa com 10,5 e 8,0 cm, respectivamente, considerando que apenas os dois últimos estavam regulados para máxima profundidade de corte. Nota-se, também, que houve eficiência nas regulagens efetuadas no campo. HOOGMOED \& DERPSCH (1985) e SCHLOSSER et al. (1992) também observaram que a grade aradora não consegue atingir grande profundidade de operação.

Resultados similares aos deste trabalho já haviam sido obtidos por COAN (1995). Observa-se a existência de uma relação entre a espessura média da camada mobilizada e a área mobilizada do solo.

Com o empolamento do solo, que representa a relação percentual entre a área de elevação e a área mobilizada resultante da ação dos órgãos ativos dos equipamentos de preparo periódico primário do solo, é possível avaliar a predisposição do solo à erosão.

Os resultados apresentados na Tabela 1 mostram haver diferença significativa entre os tratamentos avaliados. O maior empolamento ocorreu para a enxada rotativa (40,9\%), seguido pela grade aradora (24,3\%), escarificador (19,6\%), arado de discos $(18,0 \%)$ e arado de aivecas $(10,9 \%)$.

Verifica-se que o arado de aivecas, considerando-se a elevada espessura média da camada mobilizada do solo e a menor área de elevação, foi o equipamento que proporcionou o menor revolvimento do solo, predispondo-o menos à ação erosiva das chuvas. Resultados semelhantes foram obtidos no trabalho realizado por CARVALHO FILHO et al. (2001); entretanto, SALVADOR et al. (1993) não encontraram diferenças significativas entre os valores de empolamento de arados, grades e escarificadores, provavelmente, pelo fato de se tratar de ferramentas ativas diferentes dos equipamentos e das variações de algumas características operacionais, principalmente a velocidade. 
Observa-se que os índices de rugosidade eram iguais antes do preparo do solo e que, logo após a mobilização, apresentaram diferenças significativas, em função dos equipamentos empregados no preparo primário do solo (Tabela 1).

Os arados apresentaram os maiores índices de rugosidade, enquanto a enxada rotativa proporcionou o menor, fato também verificado por BURWELL \& LARSON (1969).

O escarificador, novamente, confirma-se como equipamento adequado ao sistema conservacionista por proporcionar, como os arados, elevado índice de rugosidade, o que, de acordo com COAN (1995) e CARVALHO FILHO et al. (2001), gera menor tendência de formação de encrostamento superficial, aumentando e influenciando na quantidade de água que poderá ficar retida nas depressões, retardando o início de escoamento superficial, em caso de chuvas. COAN (1995), apesar de não ter observado diferenças significativas trabalhando com equipamentos semelhantes, também destaca que os maiores índices foram obtidos com uso de arado de aivecas, arado de discos e escarificador.

DALLMEYER et al. (1989) observaram que diferentes órgãos ativos podem ocasionar valores similares de índice de rugosidade, o que, aplicado à seleção de equipamentos, pode trazer vantagens econômicas e de conservação do solo e da água. Os mesmos autores consideram que podem ser obtidos valores distintos de índice de rugosidade, em função da metodologia adotada na determinação dessa variável, e que, portanto, cumpre observar esse detalhe para evitar a discussão dos valores numéricos absolutos em comparação.

Quanto à modificação da rugosidade do solo (Tabela 1), nota-se que, apesar de não haver diferenças significativas para os equipamentos analisados, os maiores valores de modificação da rugosidade do solo obtidos, em valores absolutos, foram, respectivamente, para o arado de discos, arado de aivecas, escarificador, grade aradora e finalmente, para a enxada rotativa. Considerandose que o coeficiente de variação foi muito elevado $($ C.V. $=59,1 \%)$, embora se tenha tomado cuidado na obtenção dos valores analisados, confirma-se a necessidade de mais estudos para a melhoria de uma metodologia adequada para interpretação técnica eficiente.

\section{CONCLUSÕES}

A enxada rotativa, nas condições em que foi regulada, ocasionou elevado empolamento e baixo índice de rugosidade, caracterizando-se como o equipamento que proporcionou a maior desagregação do solo.

O escarificador promoveu elevado índice de rugosidade e baixo empolamento, o que atende à condição conservacionista.

Há necessidade de melhoria da metodologia na determinação dos índices de rugosidade e da modificação da rugosidade.

\section{REFERÊNCIAS}

ALLMARAS, R.R.; BURWELL, R.E.; LARSON, W.E.; HOLT, R.F.; NELSON, W.W. Total porosity and randon roughness of the interrow zone as influenced by tillage. Washington: USDA, 1966. 22 p. (Conservation Research Report, 7).

ASAE. AMERICAN SOCIETY OF AGRICULTURAL ENGINEERS. Terminology and definitions for soil tillage and soil tool relationships. In: ASAE Standards 1982. St. Joseph, 1982. p.229-41. (ASAE EP291.1)

BAUDER, J.W.; RANDAL, G.W.; SWAN, J.B. Effect of four continuous tillage system on mechanical impedance of a clay loam soil. Soil Science Society of America Journal, Madison, v.45, p.802-6, 1981.

BERTOL, I.; AMARAL, A.J.; VÁSQUEZ, E.V.; GONZÁLES, A.P.; BARBOSA, F.T.; BRIGNONI, L.F. Relações da rugosidade superficial do solo com o volume de chuva e com 
estabilidade de agregados em água. Revista Brasileira de Ciência do Solo, Viçosa, v.30, p.543-53, 2006.

BUHLER, D.D. Influence of tillage systems on weed population dynamics and management in corn and soybean in the central USA. Crop Science, Madison, v.35, n.5, p.1247-58, 1995.

BURWELL, R.E.; LARSON, W.E. Infiltration as influenced by tillage - induces random roughness and porespace. Soil Science American Proceedings, Madison, v.33, n.3, p.449-452, 1969.

CARVALHO FILHO, A.; CENTURION, J.F.; SILVA, R.P.; TEIXEIRA, D.S.; REQUI, E.; HOLLMANN, G. Rugosidade do solo sob diferentes sistemas de preparo. In: CONGRESSO BRASILEIRO DE CIÊNCIA DO SOLO, 28., 2001. Londrina. Anais... Londrina: Sociedade Brasileira de Ciência do Solo, 2001. p.248.

CASTRO, L.G.; COGO, N.P.; VOLK, L.B.S. Alterações na rugosidade superficial do solo pelo preparo e pela chuva e sua relação com a erosão hídrica. Revista Brasileira de Ciência do Solo, Viçosa, v.30, n.2, p.339-52, 2006.

COAN, O. Sistemas de preparo de solo: efeitos sobre a camada mobilizada e no comportamento das culturas do feijoeiro (Phaseolus vulgaris L.) e do milho (Zea mays L.), conduzidas em rotação. 1995. 138 f. Tese (Livre-Docência) - Faculdade de Ciências Agrárias e Veterinárias, Universidade Estadual Paulista, Jaboticabal, 1995.

DALLMEYER, A.U. Eficiência energética e operacional de equipamentos conjugados de preparo de solo. 1994. 157 f. Tese (Doutorado em Energia na Agricultura) - Faculdade de Ciências Agronômicas, Universidade Estadual Paulista, Botucatu, 1994.

DALLMEYER, A.U.; SALVADOR, N.; LAGE, G.; FERRAUDO, A.S.; GAMERO, C.A. Avaliação da rugosidade do solo, sob doze tipos de preparo em Latossolo Roxo Álico. In: CONGRESSO BRASILEIRO DE ENGENHARIA AGRÍCOLA, 18., 1989. Recife, Anais... Jaboticabal: SBEA, 1989. v.1, p.268-82.

DANIEL, L.A.; MARETTI, H.J. Avaliação de camada de solo compactado e análise de crescimento de plantas. In: SILVEIRA, G.M. IV Ciclo de estudos sobre Mecanização Agrícola. Campinas: Fundação Cargill, 1990. p.22-38.

DERPSCH, R.; ROTH, C.H.; SIDIRAS, N.; KOPKE, U. Controle da erosão no Paraná, Brasil: sistemas de cobertura do solo, plantio direto e preparo no conservacionismo do solo. Eschborn: GTZ, 1991. 272 p.

EMBRAPA.EMPRESA BRASILEIRA DE PESQUISA AGROPECUÁRIA. Centro Nacional de Pesquisa dos solos. Sistema brasileiro de classificação dos solos. Brasília, 1999. 412 p.

FURLANI, C.E.A. Efeito do preparo do solo e da cobertura de inverno na cultura do feijoeiro (Phaseolus vulgaris L.). 2000. 218 f. Tese (Doutorado em Energia na Agricultura) - Faculdade de Ciências Agronômicas, Universidade Estadual Paulista, Botucatu, 2000.

GAMERO, C.A.; BENEZ, S.H. Avaliação da condição do solo após a operação de preparo. In: SILVEIRA, G.M. IV Ciclo de estudos sobre mecanização agrícola. Jundiaí: Fundação Cargill, 1990. p.12-21.

GROHMANN, F.; ARRUDA, H.V. Influência do preparo do solo sobre a estrutura da Terra Roxa Legítima. Bragantia, Piracicaba, v.20, p.1203-9, 1961.

HOOGMOED, W.B.; DERPSCH, R. Chisel ploughing as an alternative tillage system in Paraná, Brazil. Soil and Tillage Research, Amsterdam, v.6, n.1, p.53-67, 1985.

KAMPHORST, E.C.; JETTEN, V.; GUÉRIF, J.; PITKÄNEN, J.; IVERSEN, B.V.; DOUGLAS, J.T.; PAZ, A. Predicting depressional storage from soil surface roughness. Soil Society America Journal, Madison, v.64, n.5, p.1749-58, 2000. 
OLIVEIRA, A.D. Arado de discos: desempenho em função do ângulo do apo e das condições de superfície de um solo Podzólico Vermelho-Amarelo Câmbico fase Terraço. 1997. Dissertação (Mestrado em Mecanização Agrícola) - Universidade Federal de Viçosa, Viçosa, 1997.

SALVADOR, N.; BENEZ, S.H.; BICUDO, S.J. Preparo periódico do solo I: desempenho operacional e mobilização do solo. In: CONGRESSO BRASILEIRO DE ENGENHARIA AGRÍCOLA, 22., 1993, Ilhéus. Anais... Ilhéus: CEPLAC/SBEA, 1993. v.3, p.1710-20.

SANTOS. J.E.G. Equipamento conjugado de preparo do solo: desempenho em função da posição das hastes escarificadoras, do tipo de ponteira e da rotação do rotor. 1993. $178 \mathrm{f}$. Tese (Doutorado em Energia na Agricultura) - Faculdade de Ciências Agronômicas, Universidade Estadual Paulista, Botucatu, 1993.

SCHLOSSER, J.F.; SILVEIRA, D.R. da; KOCHHANN, M.R.; CARGNELUTTI FILHO, A. Preparo do solo para o arroz (Oriza sativa L.) irrigado: desempenho operacional dos implementos. In: CONGRESSO BRASILEIRO DE ENGENHARIA AGRÍCOLA, 21., 1992. Santa Maria. Anais... Santa Maria: SBEA, 1992. v.3, p.1521-33.

SCHICK, J.; BERTOL, I.; BATISTELA, O.; BALBINOT JÚNIOR; A.A. Erosão hídrica em Cambissolo Húmico alumínico submetido a diferentes sistemas de preparo e cultivo. Revista Brasileira de Ciência do Solo, Viçosa, v.24, n.3, p.427-36, 2000.

SILVA, L.G.; GAMERO, C.A. Efeitos de ordens de gradagens e de sistemas de aração sobre o desenvolvimento e produção do feijoeiro. In: CONGRESSO BRASILEIRO DE ENGENHARIA AGRÍCOLA, 22., 1993, Ilhéus. Anais... Ilhéus: Sociedade Brasileira de Engenharia Agrícola, 1993. v.3, p.1520-35. 